\title{
Temporal Resolution of Misfolded Prion Protein Transport, Accumulation, Glial Activation, and Neuronal Death in the Retinas of Mice Inoculated with Scrapie
}

\author{
M. Heather West Greenlee, ${ }^{\dagger}{ }^{\dagger}$ Melissa Lind, ${ }^{*}$ Robyn Kokemuller, ${ }^{\dagger}$ Najiba Mammadova ${ }^{\dagger}$ Naveen Kondru, ${ }^{*}$ Sireesha Manne, ${ }^{*}$ \\ Jodi Smith, ${ }^{\dagger}$ Anumantha Kanthasamy, ${ }^{*}$ and Justin Greenlee
}

From the Department of Biomedical Sciences, * Iowa State University College of Veterinary Medicine, Ames; and the Virus and Prion Disease Unit, ${ }^{\dagger}$ National Animal Disease Center, US Department of Agriculture, Ames, Iowa

Accepted for publication

May 23, 2016.

Address correspondence to $\mathrm{M}$. Heather West Greenlee, Ph.D., Department of Biomedical Sciences, Iowa State University College of Veterinary Medicine, 2008 Vet Med, Ames, IA 50011-1250. E-mail: mheather@iastate.edu.

\begin{abstract}
Currently, there is a lack of pathological landmarks to describe the progression of prion disease in vivo. Our goal was to use an experimental model to determine the temporal relationship between the transport of misfolded prion protein $\left(\mathrm{PrP}^{\mathrm{Sc}}\right)$ from the brain to the retina, the accumulation of $\operatorname{Pr} \mathrm{P}^{\mathrm{Sc}}$ in the retina, the response of the surrounding retinal tissue, and loss of neurons. Retinal samples from mice inoculated with RML scrapie were collected at 30,60, 90, 105, and 120 days post inoculation (dpi) or at the onset of clinical signs of disease (153 dpi). Retinal homogenates were tested for prion seeding activity. Antibody staining was used to assess accumulation of $\mathrm{PrP}^{\mathrm{Sc}}$ and the resulting response of retinal tissue. Loss of photoreceptors was used as a measure of neuronal death. $\mathrm{PrP}^{\mathrm{Sc}}$ seeding activity was first detected in all samples at $60 \mathrm{dpi}$. Accumulation of $\mathrm{PrP}^{\mathrm{Sc}}$ and coincident activation of retinal glia were first detected at $90 \mathrm{dpi}$. Activation of microglia was first detected at $105 \mathrm{dpi}$, but neuronal death was not detectable until $120 \mathrm{dpi}$. Our results demonstrate that by using the retina we can resolve the temporal separation between several key events in the pathogenesis of prion disease. (Am J Pathol 2016, 186: 2302-2309; http://dx.doi.org/10.1016/j.ajpath.2016.05.018)
\end{abstract}

Transmissible spongiform encephalopathies (TSEs) are a family of diseases caused by the accumulation of misfolded prion protein $\left(\mathrm{PrP}^{\mathrm{Sc}}\right){ }^{1}$ During progression of TSEs, like many protein misfolding disorders, transport of misfolded protein from one central nervous system (CNS) structure seeds protein misfolding and accumulation in another. ${ }^{2}$ The details underlying this series of events that begins with the arrival of misfolded protein in a CNS structure and ends with neuronal death in that structure are not well understood. Seeding the brain with an inoculum of misfolded prion protein induces TSEs; thus, these diseases provide a unique opportunity to study the transport of $\mathrm{PrP}^{\mathrm{Sc}}$ from one CNS structure to another.

Currently, there is no treatment for TSEs. Although in silico and in vitro approaches have been effective at identifying compounds with therapeutic potential, ${ }^{3-8}$ development of effective therapies would be facilitated by a well-described in vivo model of misfolded protein transport and accumulation, with objective measures of neural degeneration.

The retina is part of the CNS and is affected by numerous protein misfolding diseases, including Alzheimer disease, Parkinson disease, ${ }^{10-12}$ and numerous TSEs, including scrapie in sheep, ${ }^{13,14}$ chronic wasting disease in

\footnotetext{
Supported by US Department of Agriculture to the Virus and Prion Disease Unit at the National Animal Disease Center and an NIH T-35 grant to The Iowa State University College of Veterinary Medicine (T35-OD012199-11 to M.L.).

Mention of trade names or commercial products in this article is solely for the purpose of providing specific information and does not imply recommendation or endorsement by the US Department of Agriculture. The US Department of Agriculture is an equal opportunity provider and employer.

Disclosures: None declared.

Current address of J.S., Department of Veterinary Pathology, Iowa State University College of Veterinary Medicine, Ames, IA.
} 
cervids, ${ }^{15-17}$ bovine spongiform encephalopathy in cattle, ${ }^{18,19}$ and Creutzfeldt-Jakob disease in humans. ${ }^{20,21}$ The retina and associated visual structures provide an excellent model to study the transport of misfolded proteins from one CNS structure to another. ${ }^{22}$

Herein, we report the time course of transport of misfolded prion protein to the retina after inoculation into the brain in a mouse model of scrapie. We describe the temporal separation between the first detection of misfolded prion protein at $40 \%$ of the 153-day incubation period, the accumulation of $\mathrm{PrP}^{\mathrm{Sc}}$ at $60 \%$ of the incubation period, the activation of microglia at $70 \%$ of the incubation period, and the loss of retinal neurons at $80 \%$ of the incubation period. Thus, we provide a well-described in vivo model that can be used to study the pathogenesis of disease and to assess the potential effectiveness of therapeutic approaches against the spread and accumulation of misfolded prion protein.

\section{Materials and Methods}

\section{Ethics Statement}

The experiments were performed in accordance with the Guide for the Care and Use of Laboratory Animals. ${ }^{23}$ The protocol was approved by the Institutional Animal Care and Use Committee at the National Animal Disease Center (protocol number 3985). Sample size was determined statistically using preliminary data from clinically affected animals.

\section{Animals and Tissue Preparation}

C57B1/6 mice, aged 6 to 8 weeks, were inoculated with a brain homogenate containing mouse-adapted Rocky Mountain Laboratories (RML) scrapie via intracranial injection into the right cerebral hemisphere at the level of the temporal bone. Tissue collection occurred at 30,60, 90, 105, and 120 days post inoculation (dpi), along with another group composed of animals exhibiting clinical signs approximately 155 dpi. For each time point, there were two groups of age-matched negative control mice: a mock-inoculated group that was inoculated with brain homogenate from healthy mice and a noninoculated group that received no inoculation (five to six animals were included in each group). Globes were collected into Bouin's fixative for 24 hours and post-fixed in $10 \%$ formalin until processing. Tissues were then embedded in paraffin, divided into sections ( $4 \mu \mathrm{m}$ thick), and affixed to slides for hematoxylin and eosin staining and immunolabeling.

\section{Expression and Purification of rPrP Substrate}

Recombinant prion protein (rPrP) used in RT-QuIC assay was expressed and purified using standard protocol from previous reports. ${ }^{24,25}$ Briefly, rPrP composed of the Syrian hamster residues 90 to 231 in pET vector with N-terminal histidine sequence was transformed in to Escherichia coli Rosetta2(DE3) cells. The cultures were grown under continuous selection with kanamycin and chloramphenicol in the Lysogeny broth media. The log phase cultures were scaled up with Overnight Express Autoinduction System 1 for protein induction. Later, the inclusion bodies were isolated from bacterial pellets using BugBuster Master Mix and were denatured in $8 \mathrm{M}$ guanidine hydrochloride. Later, the $\mathrm{rPrP}$ is bound to pre-equilibrated nickel-nitrilotriacetic acid superflow resin for 50 minutes. The beads were packed in Akta \#XK26 column, and rPrP was purified using nickelnitrilotriacetic acid affinity chromatography on a Bio-Rad Duo flow system in a refrigerated room. On column refolding was performed using gradient dilution of guanidine hydrochloride over 5 hours. Pure rPrP was eluded from the column using imidazole binding with increasing linear gradient. Chilled dialysis buffer was added to the peak elude fractions, and dialyzed overnight against three changes of chilled dialysis buffer $\left(10 \mathrm{mmol} / \mathrm{L} \mathrm{NaPO}_{4}, \mathrm{pH} 5.8\right)$ after initial filtration. Protein concentration was determined using the extension coefficient and filtering through $100-\mathrm{kDa}$ cutoff filters before subjecting to RT-QuIC assay. The quality of rPrP was confirmed by NMR and CD spectral analyses.

\section{RT-QuIC Assay for Retina Samples}

The RT-QuIC assay was performed using previously published protocols from published reports ${ }^{24,26,27}$ with slight modifications. The reaction mixtures consisted of final concentrations of $350 \mathrm{mmol} / \mathrm{L} \mathrm{NaCl}, 0.1 \mathrm{mmol} / \mathrm{L}$ EDTA, $10 \mu \mathrm{mol} /$ $\mathrm{L}$ thioflavin $\mathrm{T}, 0.1 \mathrm{mg} / \mathrm{mL} \mathrm{rPrP}$, and $0.002 \%$ SDS in $1 \times$ phosphate-buffered saline. The retina samples from time course RML scrapie-infected mice were washed twice and homogenized using repeated pipetting and sonication in a cup sonicator with two pulses of 30 seconds. The clarified supernatants were normalized to protein concentration using Bradford protein assay. The reactions consisted of $5 \mu \mathrm{g}$ of protein from retina homogenates that were used as seed. We included mouse brain homogenates $(0.5 \mu \mathrm{g})$ from clinical RML scrapie infected mice, mock brain homogenates, and blank (buffer) as controls. All of the samples were run using a blinded study design (N.K., S.M., A.K.). The plates were sealed with Nalgene Nunc plate sealer and subjected to RT-QuIC assay. The plates were incubated at $42^{\circ} \mathrm{C}$ in Cytation 3 (Biotek, Winooski, VT) plate reader with alternate cycles of 1-minute shake (double orbital) and rest. All of the samples were run in quadruplicates, and samples were judged to be positive as reported previously. ${ }^{27}$ Average fluorescence readings of four replicates plus 10 times SD of negative controls were the criteria for determining the threshold. Recording of thioflavin T fluorescence ( $450 \pm 15 \mathrm{~nm}$ excitation and $480 \pm 10 \mathrm{~nm}$ emission) was noted every 30 minutes, and data analysis was performed using Biotek's Gen5 software version 2.07.17.

\section{Immunohistochemistry}

Paraffin-embedded tissues were analyzed using immunohistochemistry. After deparaffinization and rehydration, tissue 
sections were autoclaved for 20 minutes in an antigen retrieval solution (DAKO Target Retrieval Solution; Dako Corp., Carpinteria, CA). Mouse anti-PrP (6C2; CVI-WUR, Lelystad, Neth) was diluted 1:2000. Rabbit anti-glial fibrillary acidic protein (GFAP) (Dako Corp.) was diluted at 1:10,000. Slides were incubated in primary antibodies $6 \mathrm{C} 2$ overnight at $4{ }^{\circ} \mathrm{C}$ and GFAP for 24 hours at room temperature and 72 hours at $4^{\circ} \mathrm{C}$. Immunoreactivity was developed using Envision + Dual Link System-horseradish peroxidase (Dako Corp.) with diaminobenzidene (Vector Labs, Peterborough, UK), and slides were counterstained with hematoxylin. Images were captured using a Nikon DS camera on a Nikon Eclipse 50i microscope. For immunofluorescence detection of CD68 immunoreactivity (1:100; Dako Corp.), slides were incubated with Cy3 conjugated AffiniPure secondary antibodies (1:300; Jackson ImmunoResearch, West Grove, PA) for 1.5 hours and counterstained with DAPI $(1 \mu \mathrm{g} / \mathrm{mL}$; Sigma-Aldrich, St. Louis, MO). Immunofluorescence images were captured using a commercial upright microscope system (Zeiss AxioPlan 2 Microscope Imaging System; Oberkochen, Germany).

CD68 immunoreactivity was quantified using ImageJ analysis software version $1.49 \mathrm{v}$ (http://imagej.nih.gov/ij; $\mathrm{NIH}$, Bethesda, MD), and was expressed as a percentage of the total image area (outer limiting membrane to inner limiting membrane of the central retina) using constant thresholds. Values at each time point were pooled and analyzed with oneway analysis of variance and Tukey's post hoc test using Prism 6 for Windows (Graph Pad Software, San Diego, CA).

\section{Assessment of Neuronal Loss}

Thinning of the outer nuclear layer was measured by counts of cell bodies spanning the layer. For each cross section, three counts were made in the central retina and averaged into a single value for each animal. Data were analyzed with one-way analysis of variance using Dunnett's multiple comparison test.

\section{Results}

\section{Incubation Period}

Seventeen C57B1/6 mice were used to determine the incubation period of mouse-adapted (RML) scrapie. The average incubation period was $153 \mathrm{dpi}$, with a SD of 1.4 days. On the basis of a 153-day incubation period, tissues were assayed at $19.6 \%$ of total incubation (30 dpi), 39.2\% of total incubation (60 dpi), $58.8 \%$ of incubation ( $90 \mathrm{dpi}), 68.6 \%$ of incubation (105 dpi), 78.4\% of incubation (120 dpi), and $100 \%$ of incubation period (clinical illness).

\section{$\mathrm{PrP}^{\mathrm{Sc}}$ Seeding Activity Is Detected in the Retina at Approximately $40 \%$ of Incubation}

The real-time quaking induced conversion (RT-QuIC) assay was used to determine the time course of misfolded prion protein transport from the brain to the retina. Seeding activity in the RT-QuIC assay was noted in all replicates (both biological and technical) of clinical, $120 \mathrm{dpi}, 90 \mathrm{dpi}$, and 60 dpi retina samples but not in retinas from mock inoculated animals or from 30 dpi inoculated retina samples (Figure 1A). Seeding activity in retinal samples was comparable to the RML scrapie infected brain homogenate (Figure 1A). When data were expressed as amyloid formation rate [the inverse of the time for the thioflavin $\mathrm{T}$ fluorescence signals to cross the threshold (in hours)], it demonstrated that amyloid formation rate is proportional to the incubation period. That is, the longer the course of the disease, the greater the rate of amyloid formation (Figure 1B). The mean rate of amyloid formation from retina of 30,60, 90, and $120 \mathrm{dpi}$ and clinical were $0,0.06,0.12,0.17$, and 0.14 hours $^{-1}$, respectively.

\section{$\mathrm{PrP}^{\mathrm{Sc}}$ Immunoreactivity Is Detected in the Retina at} Approximately $60 \%$ of Incubation Period

Immunohistochemistry was used to assess $\mathrm{PrP}^{\mathrm{Sc}}$ accumulation in retinal sections from mice at 30,60, 90, 105, and 120 dpi and at the time clinical illness was observed (Figure 2). Mock-infected animals (collected at the same time points) were used as controls. There was no immunostaining for $\mathrm{PrP}^{\mathrm{Sc}}$ in any of the control animals (Figure 2A). Immunostaining was first observed at $90 \mathrm{dpi}$ (at approximately $60 \%$
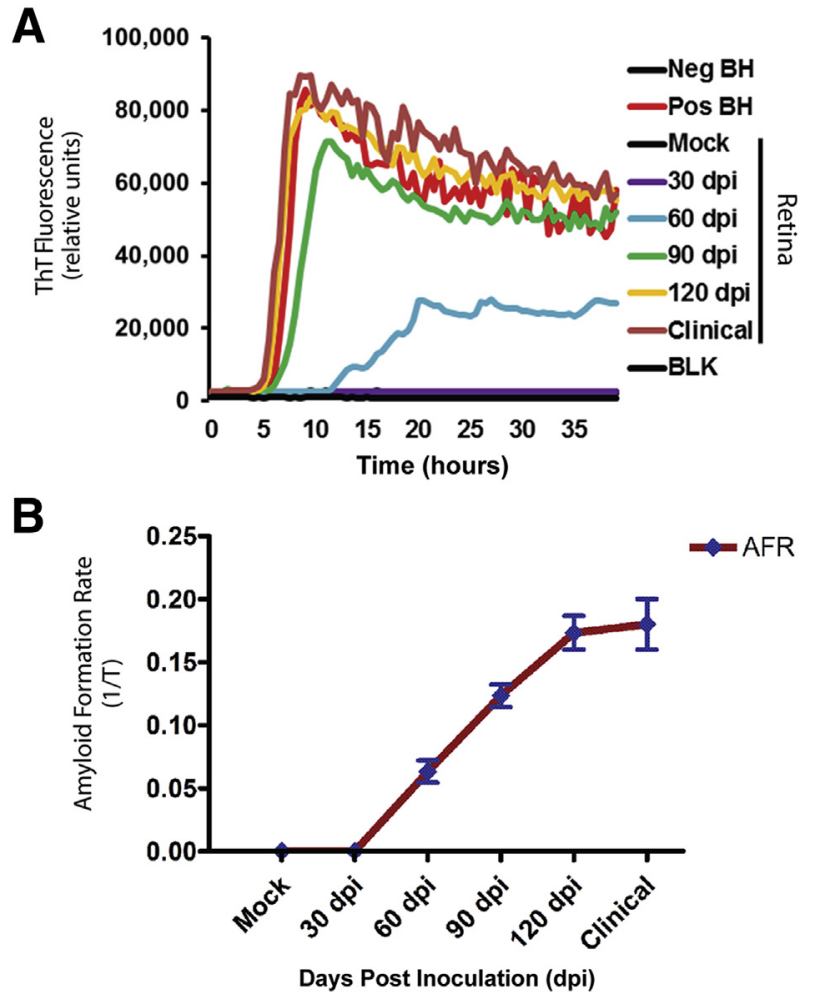

Figure $1 \quad \mathrm{PrP}^{\mathrm{Sc}}$ seeding activity in the retinas from scrapie-inoculated mice. Seeding activity is detected at $60 \mathrm{dpi}$ and all subsequent time points. A: Each trace represents average data from three biological samples, each run in quadruplicate. B: When seeding activity is expressed as amyloid formation rate (AFR), it demonstrates that amyloid formation is proportional to incubation period. BH, brain homogenate; BLK, blank well; Neg, negative; Pos, positive. 

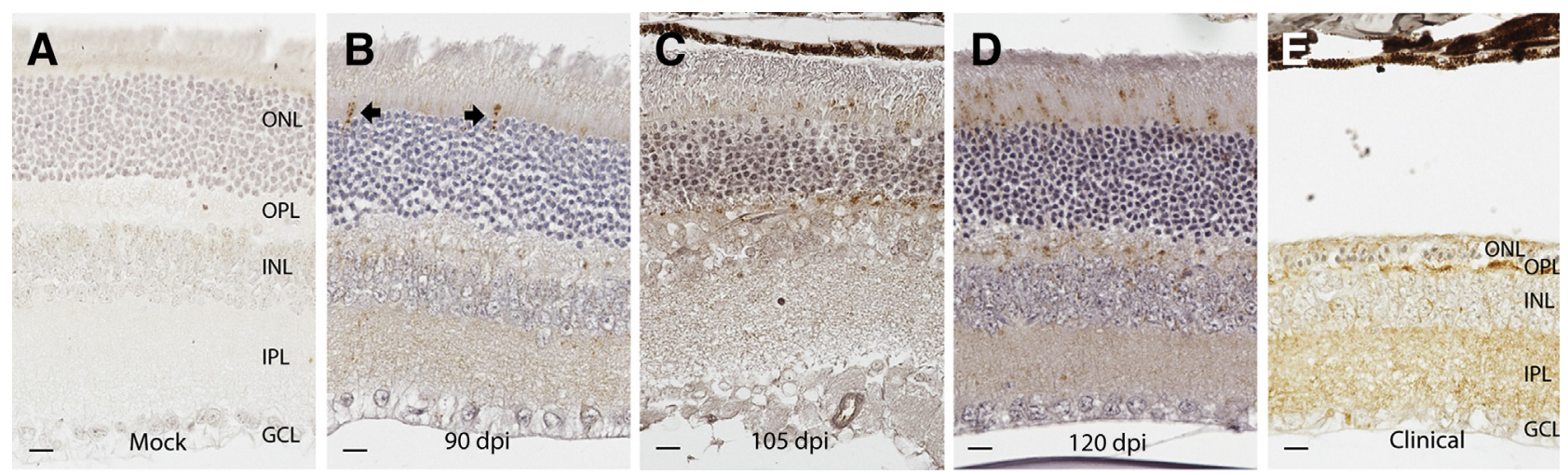

Figure $2 \mathrm{PrP} \mathrm{Sc}^{\mathrm{Sc}}$ immunoreactivity in the retinas from scrapie-inoculated mice. PrP $\mathrm{Sc}^{\mathrm{c}}$ immunoreactivity is absent from the retinas of mock-inoculated mice (A) and all mice examined before $90 \mathrm{dpi}$. B: At $90 \mathrm{dpi}$, PrP ${ }^{\mathrm{Sc}}$ immunoreactivity is observed in occasional photoreceptor inner segments in all mice examined (arrows). C: By 105 dpi, PrP ${ }^{\text {Sc }}$ immunoreactivity is consistently observed in photoreceptor inner segments and in the outer plexiform layer. D: A similar but more widespread distribution is observed at $120 \mathrm{dpi}$. E: Widespread loss of photoreceptors from the outer nuclear layer (ONL) is observed in the retinas from mice with clinical disease. In these mice, $\mathrm{PrPS}$ immunoreactivity is observed primarily in the outer plexiform layer (OPL) and inner plexiform layer (IPL). GCL, ganglion cell layer; INL, inner nuclear layer. Scale bar $=10 \mu \mathrm{m}(\mathbf{A}-\mathbf{E})$.

of the total incubation period) in multifocal photoreceptor inner segments throughout the retinal sections of each mouse examined (Figure 2B). Fifteen days later, at 105 dpi, immunoreactivity in photoreceptor inner segments was more widespread, and $\mathrm{PrP}^{\mathrm{Sc}}$ immunoreactivity also was observed in the outer plexiform (synaptic) layer (Figure 2C). At 120 $\mathrm{dpi}, \mathrm{PrP}^{\mathrm{Sc}}$ immunoreactivity was observed in the inner plexiform layer in addition to the photoreceptor layer and outer plexiform layer (Figure 2D). At the time of clinical disease (approximately $153 \mathrm{dpi}$ ), $\mathrm{PrP}^{\mathrm{Sc}}$ immunoreactivity was observed in the inner and outer plexiform layers and in the photoreceptors that remained (Figure 2E) (although by this time point, most photoreceptors had been lost).

\section{Activation of Müller Glia Accompanies Early Detection of $\operatorname{PrP}^{\mathrm{Sc}}$ at $60 \%$ of the Incubation Period}

Müller glia are radial glial cells that are derived from retinal progenitor cells. ${ }^{28}$ Quiescent Müller glia are immunoreactive for GFAP in their end feet, which are in the optic fiber layer. Under conditions of retinal stress, activated Müller glia have GFAP immunoreactivity more widely distributed in their processes that span from the optic fiber layer to the outer limiting membrane. ${ }^{29}$ Immunohistochemistry with an antibody against GFAP was used to assess activation of Müller glia in retinal sections from 30,60, 90, 105, and $120 \mathrm{dpi}$ and at the stage of clinical illness (Figure 3). Mock-inoculated animals collected at each time point were used as controls. In control animals, GFAP immunoreactivity was observed in the optic fiber layer (Figure 3A) that contains astrocytes and the end feet of Müller glia. There was not an appreciable difference between GFAP immunoreactivity in mock, 30 dpi, or 60 dpi (data not shown). However, GFAP immunoreactivity at 90 dpi was observed in processes of Müller glia that extended as far as the outer plexiform layer (Figure 3B). At 105 dpi, GFAP immunoreactivity was more robust, but still only observed between the optic fiber layer and the outer plexiform layer (Figure 3C). GFAP immunoreactivity at $120 \mathrm{dpi}$ also was
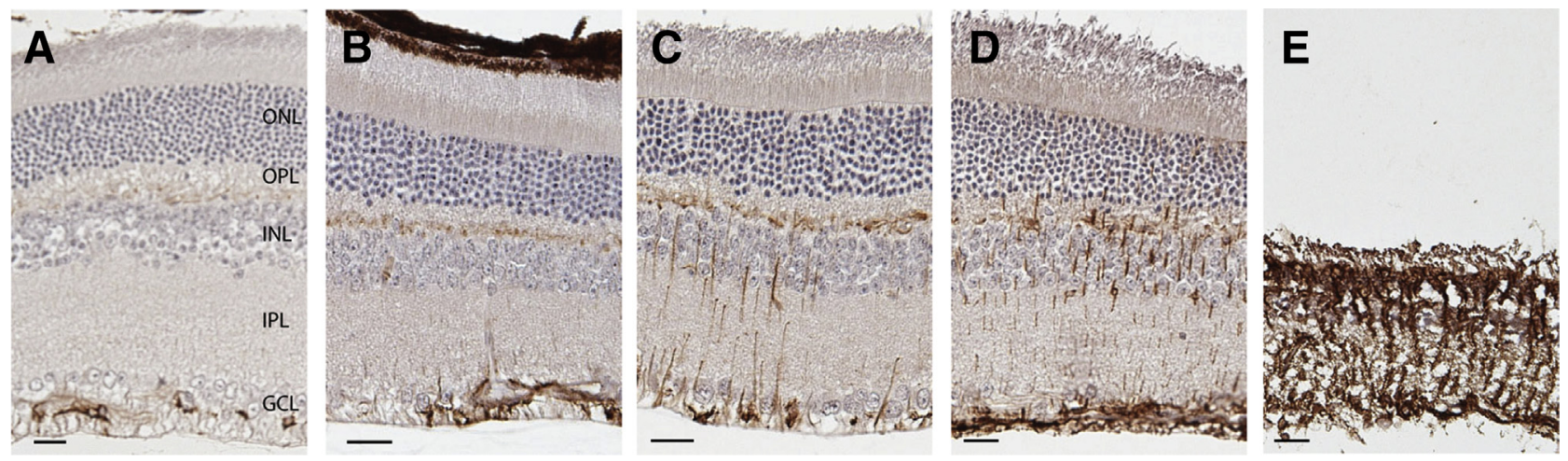

Figure 3 Activation of Müller glia in the retinas from scrapie-inoculated mice. A: In the retinas from mock-inoculated animals, glial fibrillary acidic protein (GFAP) immunoreactivity is observed only in astrocytes and Müller glial end feet in the optic fiber layer (OFL). B: GFAP immunoreactivity in other parts of Müller glia is first observed at $90 \mathrm{dpi}$ in the outer plexiform layer (OPL) and occasional processes between the OFL and OPL. GFAP immunoreactivity in Müller glia increases with incubation period as it becomes more prominent at $105 \mathrm{dpi}$ (C) and $120 \mathrm{dpi}$ (D). E: In the retinas from animals with clinical disease, GFAPimmunoreactive Müller glia span the entire extent of the retina. GCL, ganglion cell layer; INL, inner nuclear layer; IPL, inner plexiform layer; ONL, outer nuclear layer; $\mathrm{OPL}$, outer plexiform layer. Scale bar $=10 \mu \mathrm{m}(\mathbf{A}-\mathbf{E})$. 

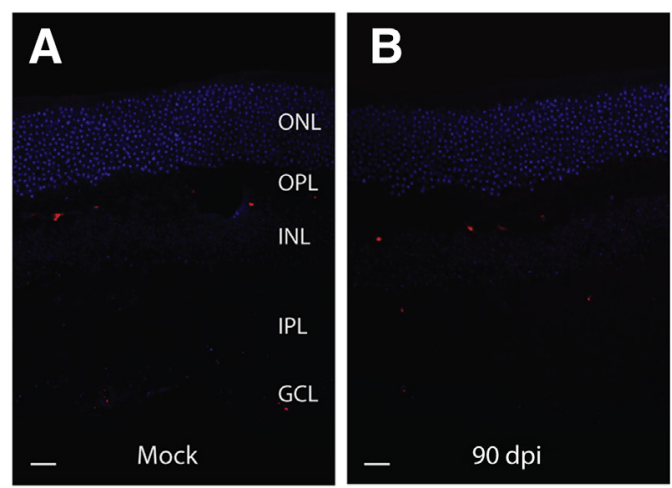

$\mathbf{F}$

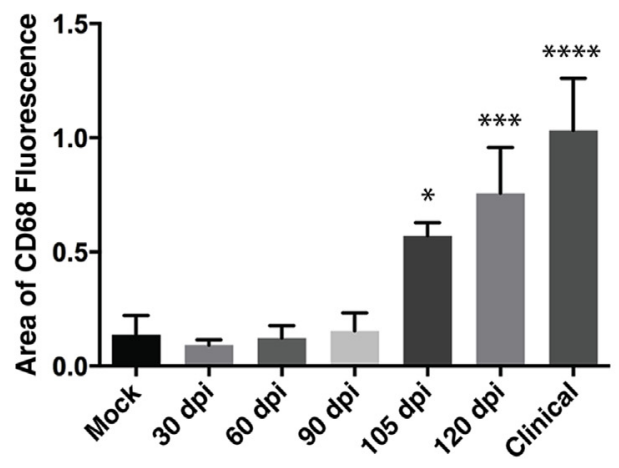

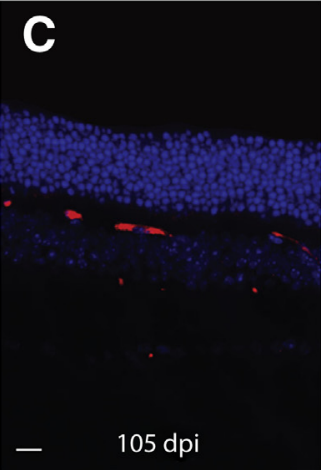
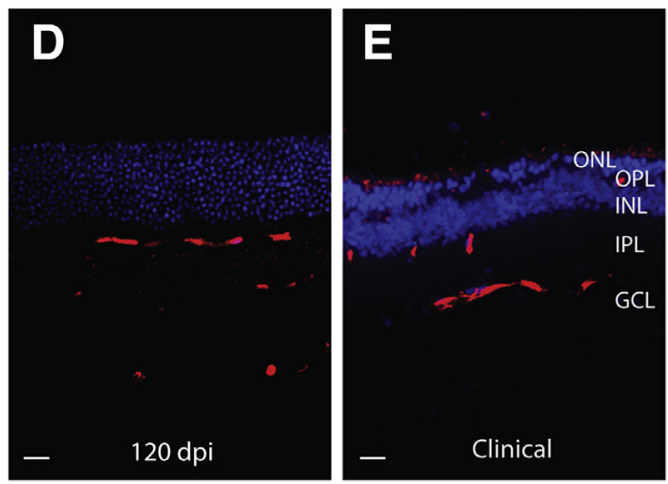

Figure 4 Activation of microglia in retinas from scrapie-inoculated mice. CD68 immunoreactivity was used to assess microglial activation. CD68 immunoreactivity is barely detectable in mock-inoculated animals (A) or retinas from animals at $90 \mathrm{dpi}$ (B). C: There is an observed increase in CD68 immunoreactivity at 105 dpi that is localized primarily to the outer plexiform layer (OPL). CD68 immunoreactivity increases at $120 \mathrm{dpi}$ (D) and in clinical retinas (E), where it is observed in both the outer and inner plexiform layers as well as the optic fiber layer. F: Quantification of CD68 immunoreactivity illustrates the increase that was first detected at $105 \mathrm{dpi}$, is greater at $120 \mathrm{dpi}$, and greatest in retinas from clinically ill animals. Data are presented as means \pm SD (F). ${ }^{*} P<0.05,{ }^{* * *} P<0.001$, and ${ }^{* * * *} P<0.0001$ versus mock. GCL, ganglion cell layer; IPL, inner plexiform layer; INL, inner nuclear layer; ONL, outer nuclear layer. Scale bar $=10 \mu \mathrm{m}(\mathbf{A}-\mathbf{E})$. observed in the outer nuclear layer and reached the outer limiting membrane in some areas (Figure 3D). At the clinical stage of disease, there was severe loss of photoreceptors and Müller glia hypertrophy with GFAP immunoreactivity observed throughout the thickness of the retina (Figure 3E).

\section{Activation of Microglia Is Detected in the Retina at $70 \%$ of Incubation Period}

An antibody against CD68 was used to assess activation of microglia in retinal sections from mice at dpi 30, 60, 90, 105,120 , and the clinical stage of disease in comparison to mock-inoculated control animals. Rare, multifocal CD68 immunoreactivity was observed in the outer plexiform layer of control retinal sections (Figure 4A). CD68 immunoreactivity in retinal sections from mice examined at 30 or 60 dpi (data not shown) or 90 dpi (Figure 4B) was not appreciably different from controls. However, at $105 \mathrm{dpi}$, CD68 immunoreactivity was more intense and widespread in the outer plexiform layer and also was observed multifocally in the inner plexiform layer and the optic fiber layer (Figure 4C). Immunoreactivity was further increased at 120 dpi (Figure 4D). In retinal sections from clinically ill mice, CD68 immunoreactivity was most intense in the optic fiber layer and the inner plexiform layer (Figure 4E). When CD68 immunoreactivity was quantified at each time point, it illustrated that the first significant increase in CD68 immunoreactivity compared to control animals was at the 105 dpi time point $(P<0.05)$ (Figure $4 \mathrm{~F})$ or at approximately $70 \%$ of incubation period.

\section{Neuronal Death Does Not Occur until $80 \%$ of Incubation Period}

To determine the time course of photoreceptor loss during RML scrapie incubation, outer nuclear layer thickness (as measured by number of photoreceptor cell nuclei) was assessed at 30,60,90,105, and $120 \mathrm{dpi}$ and in the retinas from animals with clinical disease (Figure 5). In noninoculated control animals and mock-inoculated animals, the mean outer nuclear layer thickness was 11.05 cell nuclei $( \pm 0.81)$. The mean outer nuclear layer thickness was not appreciably different from controls at 30,60, 90, or $105 \mathrm{dpi}$. At $120 \mathrm{dpi}$, however, the average outer nuclear layer thickness was significantly decreased to $8.94( \pm 1.00)$ cell nuclei $(P<0.01)$ and decreased to an average of 2.08 $( \pm 0.50)$ cell nuclei thick in retinas from animals with clinical disease. Thus, neuronal death in the form of significant photoreceptor loss $(P<0.01)$ was not detected in the retinas until approximately $80 \%$ of total incubation period.

\section{Discussion}

\section{Prion Seeding Activity Is Detected in the Retina Relatively Early in Disease Incubation}

Our results demonstrate seeding activity by RT-QuIC in the retina at $60 \mathrm{dpi}$ (approximately $40 \%$ of incubation period) in all samples assayed. Prion seeding activity in the retina was detected 30 days before the detection of $\operatorname{PrP}^{\mathrm{Sc}}$ via immunohistochemistry. This is consistent with a report that 


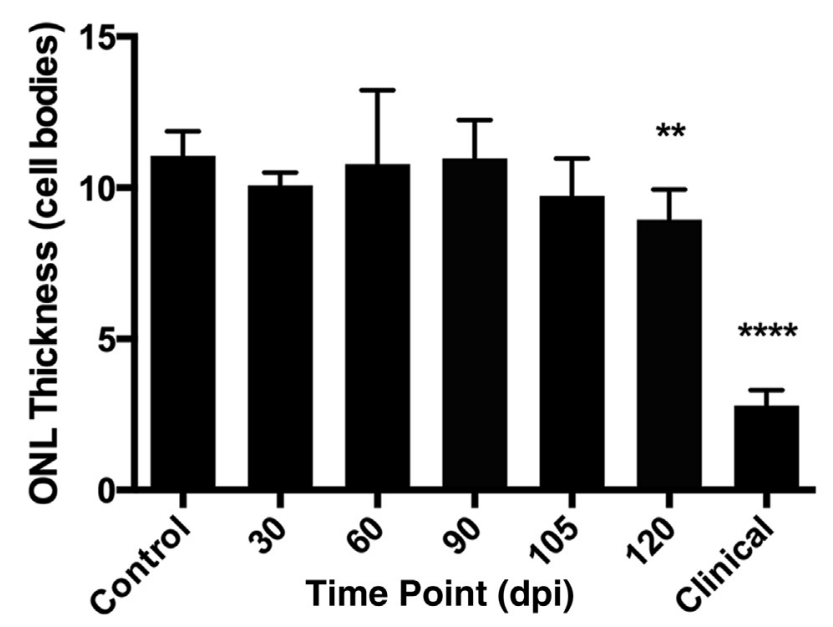

Figure 5 Thickness of outer nuclear layer in scrapie-inoculated mice. Outer nuclear layer (ONL) thickness is expressed as number of cell bodies spanning the thickness of the layer. Outer nuclear layer thickness is not different from mock-inoculated controls at 30,60, 90, or 105 dpi. Outer nuclear layer thickness decreases in the retinas at $120 \mathrm{dpi}$ and the retinas from animals with clinical illness. Data are presented as means \pm SD. ${ }^{* *} P<0.01,{ }^{* * * *} P<0.0001$ versus control.

compared RT-QuIC with immunohistochemistry and found RT-QuIC could detect prion seeding activity in tissues that were negative by immunohistochemistry. ${ }^{30}$ In the current study, the next earliest time point was $30 \mathrm{dpi}$; thus, arrival of misfolded prion protein was between 30 and 60 dpi. Because the retina is spatially distinct from the brain, where the inoculum was delivered, we are confident that the seeding activity detected is a result of transport, presumably retrograde transport via the optic nerve ${ }^{31}$; however, here we cannot entirely rule out transport by the circulatory system, as infectious prion protein was likely in blood of these animals before its detection in retina. ${ }^{32}$ Rapid retrograde transport of $\mathrm{PrP}^{\mathrm{Sc}}$ in motor tracts, on the order of 6 weeks, has been previously reported after inoculation of the sciatic nerve. ${ }^{33}$ The consistent appearance of prion seeding activity in retinas at 60 dpi may suggest widespread distribution, albeit at a lower level than detected by traditional diagnostic methods, of misfolded prion protein at a relatively early stage of the disease process. The time course of some events (eg, $\mathrm{PrP}^{\mathrm{Sc}}$ immunoreactivity) that we have reported in the retina is delayed by approximately 30 days compared to what has been reported in the brains of mice infected with RML scrapie. ${ }^{34}$

\section{Accumulation of $\operatorname{PrP}^{\mathrm{Sc}}$ Is Coincident with Activation or Retinal Glia}

We demonstrate detectable accumulation of $\operatorname{PrP}^{\mathrm{Sc}}$ at 90 dpi. Interestingly, the earliest detectable accumulation of $\mathrm{PrP}^{\mathrm{Sc}}$ was in the photoreceptor inner segments, rather than in retinal ganglion cells, whose axons comprise the optic nerve. Photoreceptors are at least two synaptic contacts removed from retinal ganglion cells, perhaps suggesting that accumulation of $\mathrm{PrP}^{\mathrm{Sc}}$ there represents a tropism for misfolded protein accumulation in photoreceptors. The earliest detection of $\operatorname{PrP}^{\mathrm{Sc}}$ accumulation was accompanied by a mild up-regulation of GFAP in Müller glia. This observation is consistent with our previous report of specific Müller glial activation in regions of early $\operatorname{PrP}^{\mathrm{Sc}}$ accumulation in retinas of sheep with scrapie. ${ }^{13}$ Up-regulation of GFAP by Müller glia is the earliest indicator of retinal stress we have been able to detect and demonstrates a response by the retinal tissue to the presence of misfolded prion protein.

\section{Neuronal Loss Does Not Occur until Late in the Disease Incubation Period}

Herein, we report widespread loss of photoreceptors in the retinas of mice with scrapie. This result is consistent with other rodent models of TSE, including a hamster model of transmissible mink encephalopathy ${ }^{35,36}$ and mouse models of scrapie $^{37}$ or Creutzfeldt-Jakob disease. ${ }^{38}$ Our results demonstrate, however, that loss of these retinal neurons does not occur until approximately $80 \%$ of the incubation period, despite the fact that prion-seeding activity was detected in the retina at $40 \%$ of the incubation period. This observation is

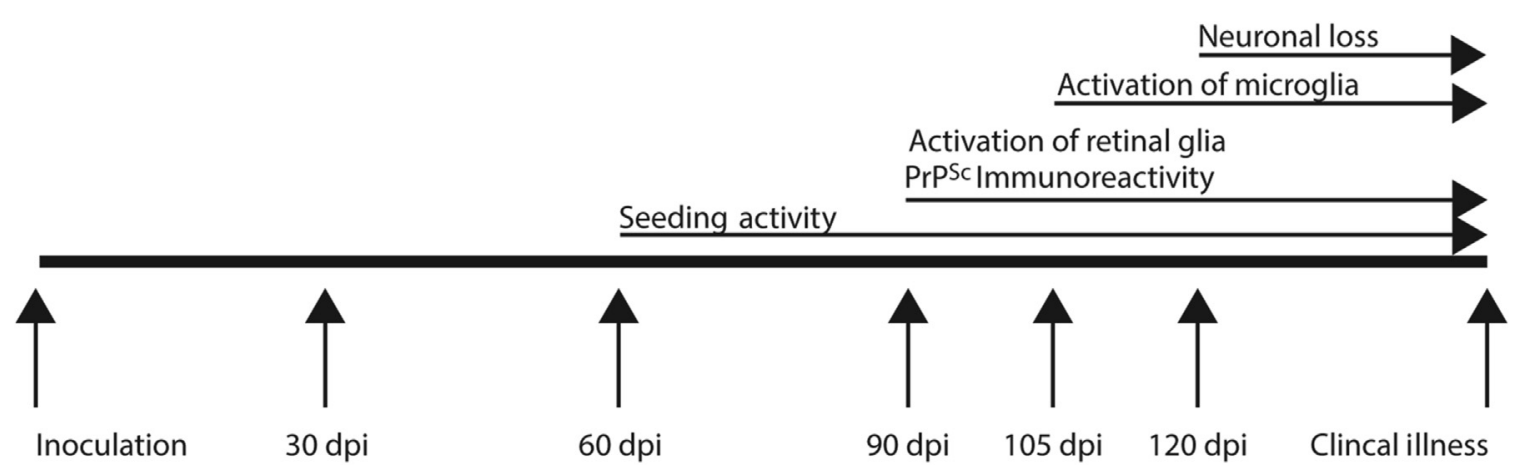

Figure 6 Summary of $\mathrm{Pr} \mathrm{P}^{\mathrm{Sc}}$ transport and scrapie pathogenesis in the mouse retina. The incubation period of RML scrapie in C57BL/6 mice in this study was 153 days. PrPS seeding activity was first observed at $60 \mathrm{dpi}$, approximately $40 \%$ of the incubation period. PrP $\mathrm{Pc}$ immunoreactivity and activation of retinal glia were first detected at $90 \mathrm{dpi}$, which is approximately $60 \%$ of the incubation period. At $105 \mathrm{dpi}$, or approximately $70 \%$ of the incubation period, activation of microglia was detected. Evidence of neuronal death in the outer nuclear layer was not detected until $120 \mathrm{dpi}$, at approximately $80 \%$ of the incubation period of RML scrapie in C57Bl/6 mice. 
consistent with prior work demonstrating an increase in prion infectivity before the development of neuropathology.

\section{Temporal Separation of Key Events in Prion Disease}

Our results demonstrate the temporal separation of several key events in the pathogenesis of prion disease beginning with the transport of misfolded prion protein from the brain to the retina (Figure 6). The sequence of events described herein is not unexpected. However, by determining the time lag between these events in vivo (eg, the lag between $\mathrm{PrP}^{\mathrm{Sc}}$ accumulation and cell death), we have provided concrete criteria that can be used not only to assess the effectiveness of anti-prion compounds ${ }^{39}$ but also to determine the stage of disease that a given compound would be most effective. For example, evaluation of an anti-prion compound in mice infected with RML scrapie between 60 and 90 dpi could provide information about the ability of the compound to attenuate accumulation of $\operatorname{PrP}^{\mathrm{Sc}}$ after misfolded prion protein has been detected in the tissue. Similarly, evaluation of an immunomodulatory or neuroprotective compound between 90 and 120 dpi could provide key information about the relationship between activated microglia and neuronal death in prion disease. ${ }^{4}$

The results reported herein contribute to a sizable body of work describing the time course of prion disease. A great deal has been done to identify early biomarkers of disease in cattle ${ }^{40-42}$ cervids ${ }^{43,44}$ primates ${ }^{45}$ sheep ${ }^{46}$ and rodent models of TSEs. ${ }^{34,47}$ The retina is a tissue that is spatially separated from the rest of the brain, has a relatively simple organization, and can be readily assessed using rapid and noninvasive techniques. This work provides a timeline for key pathologic milestones of prion disease in the retina and a model to study mechanisms of disease progression and evaluate therapeutic interventions.

\section{Acknowledgments}

We thank Kevin Hassall, David Panthen, and Trudy Tatum for providing technical support to this project.

\section{References}

1. Prusiner SB: Molecular biology of prion diseases. Science 1991, 252: $1515-1522$

2. Shearin H, Bessen RA: Axonal and transynaptic spread of prions. J Virol 2014, 88:8640-8655

3. Cashman NR, Caughey B: Prion diseases: close to effective therapy? Nat Rev Drug Discov 2004, 3:874-884

4. Zhou M, Ottenberg G, Sferrazza GF, Hubbs C, Fallahi M, Rumbaugh G, Brantley AF, Lasmezas CI: Neuronal death induced by misfolded prion protein is due to NAD + depletion and can be relieved in vitro and in vivo by NAD + replenishment. Brain 2015, 138: 992-1008

5. Cordeiro Y, Ferreira NC: New approaches for the selection and evaluation of anti-prion organic compounds. Mini Rev Med Chem 2015, 15:84-92
6. Edgeworth JA, Jackson GS, Clarke AR, Weissmann C, Collinge J: Highly sensitive, quantitative cell-based assay for prions adsorbed to solid surfaces. Proc Natl Acad Sci U S A 2009, 106:3479-3483

7. Wagner J, Ryazanov S, Leonov A, Levin J, Shi S, Schmidt F, Prix C, PanMontojo F, Bertsch U, Mitteregger-Kretzschmar G, Geissen M, Eiden M, Leidel F, Hirschberger T, Deeg AA, Krauth JJ, Zinth W, Tavan P, Pilger J, Zweckstetter M, Frank T, Bahr M, Weishaupt JH, Uhr M, Urlaub H, Teichmann U, Samwer M, Botzel K, Groschup M, Kretzschmar H, Griesinger C, Giese A: Anle138b: a novel oligomer modulator for diseasemodifying therapy of neurodegenerative diseases such as prion and Parkinson's disease. Acta Neuropathol 2013, 125:795-813

8. Hyeon JW, Choi J, Kim SY, Govindaraj RG, Jam Hwang K, Lee YS, An SS, Lee MK, Joung JY, No KT, Lee J: Discovery of novel antiprion compounds using in silico and in vitro approaches. Sci Rep 2015, 5:14944

9. Coppola G, Di Renzo A, Ziccardi L, Martelli F, Fadda A, Manni G, Barboni P, Pierelli F, Sadun AA, Parisi V: Optical coherence tomography in Alzheimer's disease: a meta-analysis. PLoS One 2015, 10: e0134750

10. Bodis-Wollner I, Kozlowski PB, Glazman S, Miri S: Alpha-synuclein in the inner retina in parkinson disease. Ann Neurol 2014, 75:964-966

11. Beach TG, Carew J, Serrano G, Adler CH, Shill HA, Sue LI, Sabbagh MN, Akiyama H, Cuenca N: Arizona Parkinson's Disease Consortium: Phosphorylated alpha-synuclein-immunoreactive retinal neuronal elements in Parkinson's disease subjects. Neurosci Lett 2014, 571:34-38

12. Chorostecki J, Seraji-Bozorgzad N, Shah A, Bao F, Bao G, George E, Gorden V, Caon C, Frohman E, Tariq Bhatti M, Khan O: Characterization of retinal architecture in Parkinson's disease. J Neurol Sci 2015, 355:44-48

13. Greenlee JJ, Hamir AN, West Greenlee MH: Abnormal prion accumulation associated with retinal pathology in experimentally inoculated scrapie-affected sheep. Vet Pathology 2006, 43:733-739

14. Regnier A, Andreoletti O, Albaric O, Gruson DC, Schelcher F, Toutain PL: Clinical, electroretinographic and histomorphometric evaluation of the retina in sheep with natural scrapie. BMC Vet Res 2011, 7:25

15. Spraker TR, O'Rourke KI, Gidlewski T, Powers JG, Greenlee JJ, Wild MA: Detection of the abnormal isoform of the prion protein associated with chronic wasting disease in the optic pathways of the brain and retina of Rocky Mountain elk (Cervus elaphus nelsoni). Vet Pathol 2010, 47:536-546

16. Keane DP, Barr DJ, Bochsler PN, Hall SM, Gidlewski T, O'Rourke KI, Spraker TR, Samuel MD: Chronic wasting disease in a Wisconsin white-tailed deer farm. J Vet Diagn Invest 2008, 20: 698-703

17. Hamir AN, Greenlee JJ, Nicholson EM, Kunkle RA, Richt JA, Miller JM, Hall M: Experimental transmission of chronic wasting disease (CWD) from elk and white-tailed deer to fallow deer by intracerebral route: final report. Can J Vet Res 2011, 75:152-156

18. Greenlee JJ, Smith JD, West Greenlee MH, Nicholson EM: Clinical and pathologic features of H-type bovine spongiform encephalopathy associated with E211K prion protein polymorphism. PLoS One 2012, $7: e 38678$

19. Greenlee MH, Smith JD, Platt EM, Juarez JR, Timms LL, Greenlee JJ: Changes in retinal function and morphology are early clinical signs of disease in cattle with bovine spongiform encephalopathy. PLoS One 2015, 10:e0119431

20. Head MW, Northcott V, Rennison K, Ritchie D, McCardle L, Bunn TJ, McLennan NF, Ironside JW, Tullo AB, Bonshek RE: Prion protein accumulation in eyes of patients with sporadic and variant Creutzfeldt-Jakob disease. Invest Ophthalmol Vis Sci 2003, 44: 342-346

21. Head MW, Peden AH, Yull HM, Ritchie DL, Bonshek RE, Tullo AB Ironside JW: Abnormal prion protein in the retina of the most commonly occurring subtype of sporadic Creutzfeldt-Jakob disease. $\mathrm{Br}$ J Ophthalmol 2005, 89:1131-1133 
22. Rahimi J, Milenkovic I, Kovacs GG: Patterns of tau and alphasynuclein pathology in the visual system. J Parkinsons Dis 2015, 5: 333-340

23. Committee for the Update of the Guide for the Care and Use of Laboratory AnimalsNational Research Council: Guide for the Care and Use of Laboratory Animals: Eighth Edition. Washington, DC, National Academies Press, 2011

24. Wilham JM, Orru CD, Bessen RA, Atarashi R, Sano K, Race B, Meade-White KD, Taubner LM, Timmes A, Caughey B: Rapid endpoint quantitation of prion seeding activity with sensitivity comparable to bioassays. PLoS Pathog 2010, 6:e1001217

25. Atarashi R, Wilham JM, Christensen L, Hughson AG, Moore RA, Johnson LM, Onwubiko HA, Priola SA, Caughey B: Simplified ultrasensitive prion detection by recombinant $\operatorname{PrP}$ conversion with shaking. Nat Methods 2008, 5:211-212

26. Haley NJ, Hoover EA: Chronic wasting disease of cervids: current knowledge and future perspectives. Annu Rev Anim Biosci 2015, 3: 305-325

27. Orru CD, Groveman BR, Hughson AG, Zanusso G, Coulthart MB, Caughey B: Rapid and sensitive RT-QuIC detection of human Creutzfeldt-Jakob disease using cerebrospinal fluid. MBio 2015, 6: e02451-14

28. Cepko C: Intrinsically different retinal progenitor cells produce specific types of progeny. Nat Rev Neurosci 2014, 15:615-627

29. Lewis GP, Fisher SK: Up-regulation of glial fibrillary acidic protein in response to retinal injury: its potential role in glial remodeling and a comparison to vimentin expression. Int Rev Cytol 2003, 230:263-290

30. Hoover CE, Davenport KA, Henderson DM, Pulscher LA, Mathiason CK, Zabel MD, Hoover EA: Detection and quantification of CWD prions in fixed paraffin embedded tissues by real-time quakinginduced conversion. Sci Rep 2016, 6:25098

31. Walis A, Liberski PP, Brown P: Ultrastructural alterations in the optic nerve in transmissible spongiform encephalopathies or prion diseases: a review. Folia Neuropathol 2004, 42(Suppl B):153-160

32. Sawyer EB, Edgeworth JA, Thomas C, Collinge J, Jackson GS: Preclinical detection of infectivity and disease-specific PrP in blood throughout the incubation period of prion disease. Sci Rep 2015, 5:17742

33. Bartz JC, Kincaid AE, Bessen RA: Retrograde transport of transmissible mink encephalopathy within descending motor tracts. J Virol 2002, 76:5759-5768

34. Sandberg MK, Al-Doujaily H, Sharps B, De Oliveira MW, Schmidt C, Richard-Londt A, Lyall S, Linehan JM, Brandner S, Wadsworth JD, Clarke AR, Collinge J: Prion neuropathology follows the accumulation of alternate prion protein isoforms after infective titre has peaked. Nat Commun 2014, 5:4347
35. Buyukmihci NC, Goehring-Harmon F, Marsh RF: Photoreceptor degeneration during infection with various strains of the scrapie agent in hamsters. Exp Neurol 1987, 97:201-206

36. Hogan RN, Bowman KA, Baringer JR, Prusiner SB: Replication of scrapie prions in hamster eyes precedes retinal degeneration. Ophthalmic Res 1986, 18:230-235

37. Foster J, Farquhar C, Fraser J, Somerville R: Immunolocalization of the prion protein in scrapie affected rodent retinas. Neurosci Lett 1999, 260:1-4

38. Hogan RN, Kingsbury DT, Baringer JR, Prusiner SB: Retinal degeneration in experimental Creutzfeldt-Jakob disease. Lab Invest 1983, 49: 708-715

39. Ghaemmaghami S, Russo M, Renslo AR: Successes and challenges in phenotype-based lead discovery for prion diseases. J Med Chem 2014, 57:6919-6929

40. Simon S, Nugier J, Morel N, Boutal H, Creminon C, Benestad SL, Andreoletti O, Lantier F, Bilheude JM, Feyssaguet M, Biacabe AG, Baron T, Grassi J: Rapid typing of transmissible spongiform encephalopathy strains with differential ELISA. Emerg Infect Dis 2008, 14: 608-616

41. Plews M, Lamoureux L, Simon SL, Graham C, Ruddat V, Czub S, Knox JD: Factors affecting the accuracy of urine-based biomarkers of BSE. Proteome Sci 2011, 9:6

42. Bannach O, Reinartz E, Henke F, Dressen F, Oelschlegel A, Kaatz M, Groschup MH, Willbold D, Riesner D, Birkmann E: Analysis of prion protein aggregates in blood and brain from pre-clinical and clinical BSE cases. Vet Microbiol 2013, 166:102-108

43. Haley NJ, Mathiason CK, Zabel MD, Telling GC, Hoover EA: Detection of sub-clinical CWD infection in conventional test-negative deer long after oral exposure to urine and feces from CWD + deer. PLoS One 2009, 4:e7990

44. Rubenstein R, Chang B, Gray P, Piltch M, Bulgin MS, SorensenMelson S, Miller MW: Prion disease detection, PMCA kinetics, and $\mathrm{IgG}$ in urine from sheep naturally/experimentally infected with scrapie and deer with preclinical/clinical chronic wasting disease. J Virol 2011, 85:9031-9038

45. Kim MO, Geschwind MD: Clinical update of Jakob-Creutzfeldt disease. Curr Opin Neurol 2015, 28:302-310

46. Batxelli-Molina I, Salvetat N, Andreoletti O, Guerrier L, Vicat G, Molina F, Mourton-Gilles C: Ovine serum biomarkers of early and late phase scrapie. BMC Vet Res 2010, 6:49

47. Lamoureux L, Simon SL, Plews M, Ruddat V, Brunet S, Graham C, Czub S, Knox JD: Urine proteins identified by two-dimensional differential gel electrophoresis facilitate the differential diagnoses of scrapie. PLoS One 2013, 8:e64044 